\title{
Dual Demodulation of Temperature and Refractive Index Using Ring Core Fiber Based Mach-Zehnder Interferometer
}

\author{
Weihao Yuan (1) and Changyuan Yu * \\ Photonic Research Centre, Department of Electronic and Information Engineering, The Hong Kong Polytechnic \\ University, Hong Kong, China; weihao.yuan@connect.polyu.hk \\ * Correspondence: changyuan.yu@polyu.edu.hk
}

check for

updates

Citation: Yuan, W.; Yu, C. Dual Demodulation of Temperature and Refractive Index Using Ring Core Fiber Based Mach-Zehnder Interferometer. Micromachines 2021, 12, 258. https://doi.org/10.3390/ mi12030258

Received: 28 January 2021

Accepted: 24 February 2021

Published: 3 March 2021

Publisher's Note: MDPI stays neutral with regard to jurisdictional claims in published maps and institutional affiliations.

Copyright: (c) 2021 by the authors. Licensee MDPI, Basel, Switzerland. This article is an open access article distributed under the terms and conditions of the Creative Commons Attribution (CC BY) license (https:// creativecommons.org/licenses/by/ $4.0 /)$.

\begin{abstract}
We report the ring core fiber spliced with single mode fiber and no core fiber which is used for temperature and refractive index (RI) sensing. The Mach-Zehnder interferometer (MZI) is formed with this kind of sandwich fiber structure and the maximum extinction ratio of the interference spectra reaches $27 \mathrm{~dB}$ with the free spectra range of $12 \mathrm{~nm}$. The MZI fiber sensor is applied for temperature sensing with the sensitivity of $69 \mathrm{pm} /{ }^{\circ} \mathrm{C}$ and $0.051 \mathrm{~dB} /{ }^{\circ} \mathrm{C}$. The RI sensitivity reaches $182.07 \mathrm{~dB} / \mathrm{RIU}$ and $-31.44 \mathrm{~nm} / \mathrm{RIU}$ with the RI ranging from 1.33 to 1.38 . The RI value can be directly demodulated with the interference dip intensity which shows insensitivity to temperature. The demodulation of temperature can be achieved by using the linear equations between dip wavelength shift with the variation of temperature and RI.
\end{abstract}

Keywords: ring core fiber; Mach-Zehnder interferometer; temperature; refractive index

\section{Introduction}

Optical fiber sensor has gained extensive attention in recent years due to its various advantages, such as low cost, ease of fabrication, compactness, good resistance to electromagnetic interference and chemical corrosion [1]. Diverse applications of fiber sensor have been researched and developed, for instance, temperature sensing [2-4], humidity sensing [5,6], refractive index (RI) sensing [7-9], strain sensing [10,11], curvature sensing [12,13], $\mathrm{pH}$ sensing [14,15], gas sensing [16-18] and so on. Temperature and RI are seemed as the important parameters because of their importance on environment monitoring, liquid quality inspection and food safety detection. Different fiber structures are studied for simultaneous measurement of temperature and RI, including Fiber Bragg Grating (FBG) [19,20], Mach-Zehnder interferometer (MZI) [21,22], Fabry-Perot interferometer (FPI) [23,24], sidepolished fiber [25] and hybrid fiber structures [26,27]. The MZI fiber sensor is regarded as one of the most effective structures. The principle of MZI sensor for temperature sensing is mainly based on the changes of effective RI difference between core and cladding and that of interference length induced by thermal effect. The alteration of effective RI difference and interference length will lead to the wavelength shift of the interference spectra, which can be used for demodulation of temperature. The RI sensing can be realized by exciting the evanescent field on the fiber surface, on which surrounding materials can make the impact on the fiber cladding modes. This can give rise to the variation of light amplitude and phase which will further result in the change of interference spectra.

In this paper, the ring core fiber (RCF) based MZI fiber sensor is proposed and experimentally demonstrated for dual demodulation of temperature and RI. The MZI sensor was fabricated by direct fusion splicing a piece of no core fiber (NCF) to a segment of RCF. Then the formed N-R fiber structure was connected to two pieces of single mode fibers (SMFs). The built SMF-NCF-RCF-SMF structure can be seemed as the miniatured MZI. Multiple fiber modes are stimulated in the RCF and combined into the SMF. The experimental results demonstrate the good interference effect of the MZI structure with the maximum extinction ratio of $27 \mathrm{~dB}$ and the free spectra range of $12 \mathrm{~nm}$. The temperature 
sensing is realized by monitoring the wavelength shift of interference dip induced by heating and cooling effect and the RI sensing can be implemented due to the adjustment of cladding modes' amplitude and phase induced by surrounding RI change. The RI value is expressed by refractive index unit (RIU). The interference dip intensity and dip wavelength shift display different responses to temperature and RI changes which makes it possible for dual demodulation of these two parameters. The variation of temperature can result in the wavelength shift with the slope of $69 \mathrm{pm} /{ }^{\circ} \mathrm{C}$ and negligible change of dip intensity in limited temperature ranges. The alteration of RI values gives rise to simultaneous wavelength shift of $-31.44 \mathrm{~nm} / \mathrm{RIU}$ and dip intensity change of $182.07 \mathrm{~dB} / \mathrm{RIU}$. Thus, the RI can be demodulated by the change of interference intensity and temperature sensing can be achieved through the linear relationship between dip wavelength with the value of temperature and RI.

\section{Experiment and Analysis}

The MZI was fabricated with four segments which consisted of double commercial single-mode fibers (SMF-28, Corning, NY, USA), no core fiber and ring core fiber. The NCF and RCF were purchased from YOFC and the diameters of SMF, NCF and RCF are the same at $125 \mu \mathrm{m}$ which enables the direct fusion splicing without diameter mismatching. The lengths of applied NCF and RCF are $1 \mathrm{~mm}$ and $20 \mathrm{~mm}$, respectively. As can be seen from Figure 1a, the cross section of RCF can be divided into three parts: fiber center (FC), ring core $(\mathrm{RC})$ and cladding area. The radii of $\mathrm{FC}, \mathrm{RC}$ and cladding are $5.45 \mu \mathrm{m}, 9.35 \mu \mathrm{m}$ and $62.5 \mu \mathrm{m}$, separately. Figure $1 \mathrm{~b}$ displays the RI distribution along the fiber diameter (yellow dash line) and the RI of RC is 0.0134 larger than that of FC and cladding with the value of 1.44402 .

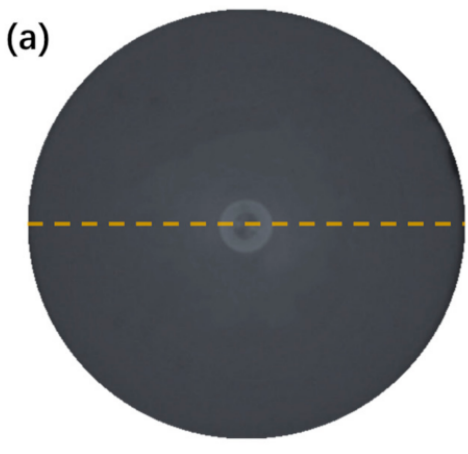

(b)

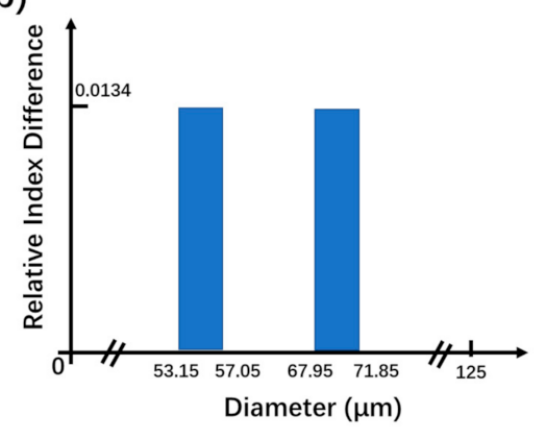

Figure 1. The microscope images of RCF (a) and the RI distribution along the diameter (dash line) (b).

Figure 2 depicts the schematic diagram of the MZI with the SMF-NCF-RCF-SMF structure. The blue parts show the SMFs with the diameter of $125 \mu \mathrm{m}$ and core diameter of $\sim 8.5 \mu \mathrm{m}$. The grey part represents the NCF with the diameter of $125 \mu \mathrm{m}$, which has the same RI value with that of SMF cladding. The yellow part depicts the RCF and the details are shown in Figure 1. The lengths of applied NCF and RCF are $1 \mathrm{~mm}$ and $20 \mathrm{~mm}$, respectively. Broadband Source (BBS) with the bandwidth from $1470 \mathrm{~nm}$ to $1670 \mathrm{~nm}$ is used as the light source. The light transmitted in SMF will firstly be coupled into NCF and then be transmitted to RCF in which multiple fiber modes will be excited including FC mode, RC mode and cladding modes. Different fiber modes will interfere and be coupled in SMF again at the splicing node of RCF and SMF. The MZI sensor is placed in heating oven for temperature sensing and the liquids with different sugar concentration are applied for RI sensing. The RI values of different liquids are measured and labeled using commercial saccharimeter. The optical spectrum analyzer (OSA, AQ6370D, YOKOGAWA, Japan) with the measuring range of $600-1700 \mathrm{~nm}$ is utilized for spectra recording. 


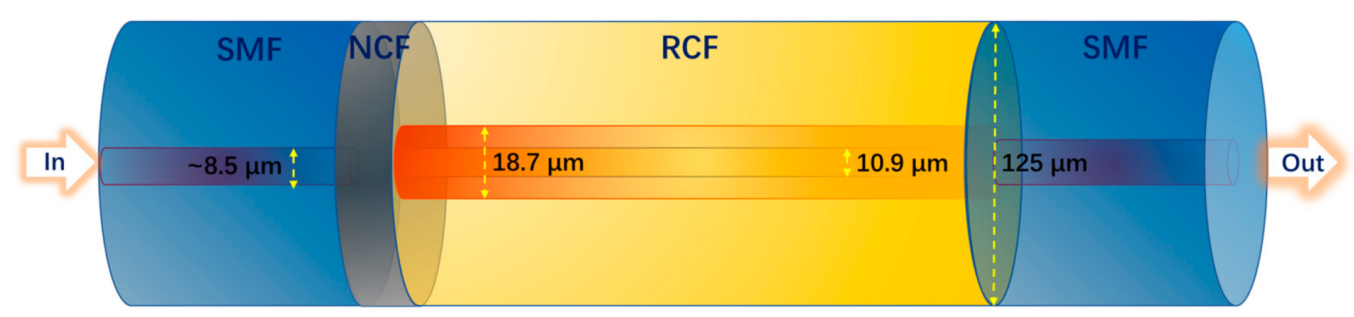

Figure 2. The schematic diagram of the MZI with the SMF-NCF-RCF-SMF structure.

To study the effect of NCF on the MZI structure, the Rsoft simulation work was done and the results were shown in Figure 3. The modeling of fiber structures with SMF-RCFSMF and SMF-NCF-RCF-SMF was conducted with the simulation tool of BeamPROP. For SMF, the diameters of core and cladding were set to be $8.5 \mu \mathrm{m}$ and $125 \mu \mathrm{m}$ with the RI values of 1.4504 and 1.4447, respectively. The lengths of NCF and RCF were built as $1 \mathrm{~mm}$ and $20 \mathrm{~mm}$. The RCF were modeled under the measurement results shown in Figure 1. The wavelength of $1550 \mathrm{~nm}$ was chosen as the free space wavelength and the grid sizes on different axes of $X, Y$ and $Z$ were set to be $0.2 \mu \mathrm{m}$. As can be seen from Figure 3a, the light is coupled from SMF to RCF and the mismatched interface between SMF and RCF gives rise to the energy redistribution which excites multiple modes in RCF including the FC mode, RC mode and cladding modes. The light energy is mainly coupled in FC mode and RC mode which induces the relatively weak cladding modes. By applying the $\mathrm{NCF}$, as shown in Figure $3 \mathrm{~b}$, the cladding modes are enhanced and RC mode becomes weaker which means that the NCF results in the redistribution of light energy among FC mode, RC mode and cladding modes. More cladding modes are excited in RCF which can significantly enhance RI sensitivity due to the strong interaction of cladding modes and surrounding materials. The Rsoft simulation results indicate that the MZI based on SMF-NCF-RCF-SMF can potentially serve as preferable sensor structure on RI sensing compared to that based on SMF-RCF-SMF structure.

$(a) \times 10^{\circ}$

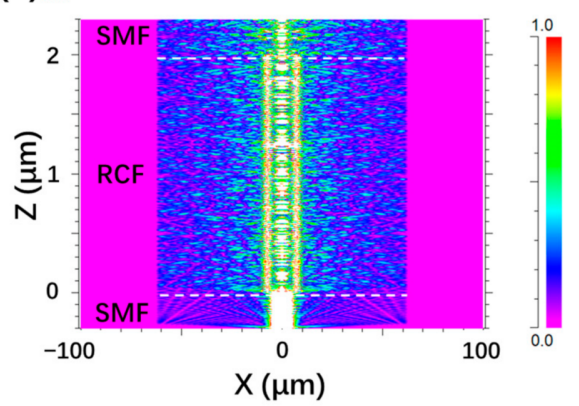

(b) $\times 10^{4}$

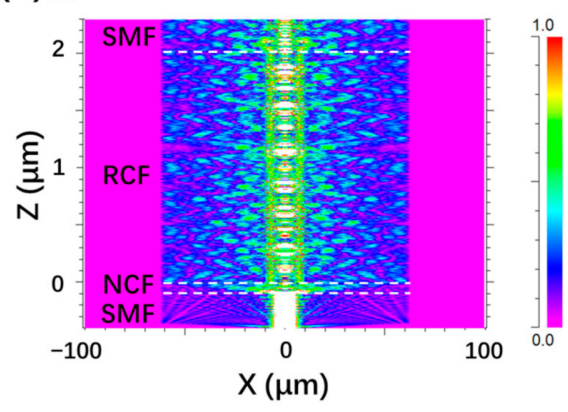

Figure 3. The Rsoft simulation results of SMF-RCF-SMF structure (a) and SMF-NCF-RCF-SMF structure $(\mathbf{b})$.

The interference spectrum of the MZI sensor based on SMF-NCF-RCF-SMF structure is displayed in Figure 4 (inset). No distinct interference pattern can be recorded if the NCF is removed from the fiber structure. The spectrum is recorded from $1500 \mathrm{~nm}$ to $1600 \mathrm{~nm}$ using OSA with the resolution of $0.02 \mathrm{~nm}$. The maximum extinction ratio (ER) is measured to be $27 \mathrm{~dB}$ and free spectral range (FSR) is $12 \mathrm{~nm}$. The large ER and FSR enable the demodulation of environmental parameters by utilizing both dip wavelength and intensity. The fast Fourier transform (FFT) is conducted and the FFT spectrum is shown in Figure 4. Except for the fundamental mode, multiple high-order modes are also excited which is in correspondence with the simulation results depicted in Figure 3b. The relationship between 
interference spectra variation and the external environment change can be explained by the interference equations below:

$$
I=I_{1}+\sum_{k} I_{2}^{k}+\sum_{m} 2 \cdot \sqrt{I_{1} \cdot I_{2}^{k}} \cos \left[\frac{2 \pi}{\lambda} \cdot\left(n_{e f f}^{1}-n_{e f f}^{2, k}\right) \cdot L\right]
$$

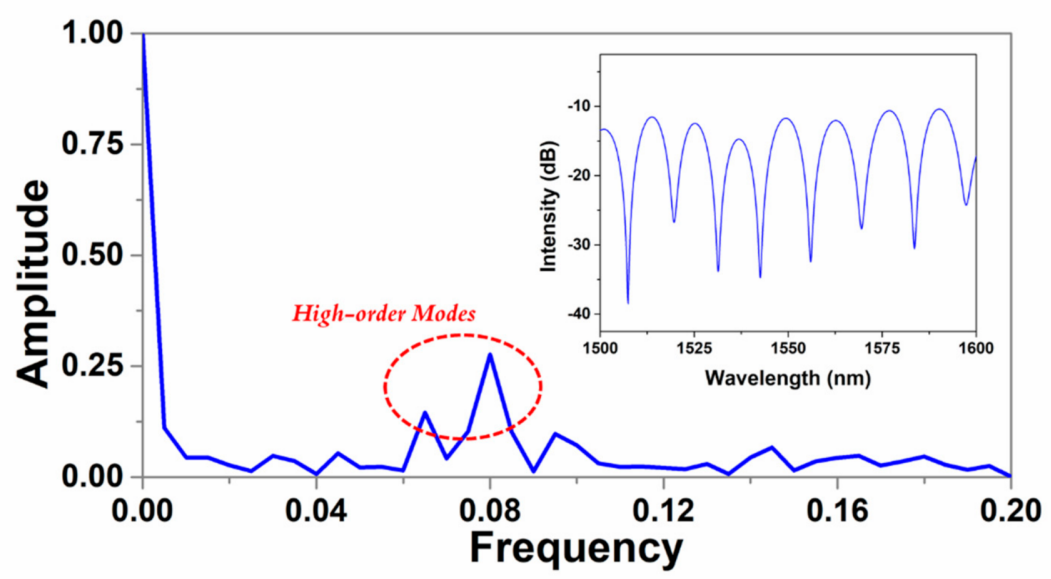

Figure 4. The interference spectrum of the MZI (inset) and the FFT spectrum.

$I, I_{1}$ represent the intensity of output light and RC mode. $I_{2}^{k}$ means the intensity of FC mode and multiple cladding modes. $n_{\text {eff }}^{1}$ represents the effective RI of the RC mode and $n_{\text {eff }}^{2, k}$ shows that of the FC mode and cladding modes. $L$ is the interference length which can be seemed as the length of the RCF. The condition of destructive interference can be explained with the following equations:

$$
\begin{gathered}
2 \pi \cdot\left(n_{\text {eff }}^{1}-n_{\text {eff }}^{2, k}\right) \cdot L / \lambda=(2 m+1) \pi \\
\lambda=\frac{2 \Delta n_{\mathrm{eff}} L}{2 m+1}
\end{gathered}
$$

$\Delta n_{\text {eff }}$ means the RI difference between the RC with the FC and the cladding. $\lambda$ in Equation (3) means the wavelength of the interference dips.

Figure 5 depicts the sensing performance of the RCF based MZI at different temperatures ranging from $35{ }^{\circ} \mathrm{C}$ to $70{ }^{\circ} \mathrm{C}$ with the interval of $5{ }^{\circ} \mathrm{C}$. The experiment was conducted in the air with surrounding RI of 1.0. As displayed in Figure 5a, the wavelength of the interference dip shows continuous shift with temperature change which can be attributed to the alteration of effective RI difference $\left(\Delta n_{\text {eff }}\right)$ and the interference length $(L)$ induced by thermal effect. The thermal-optic coefficient of Ge-doped silica core is larger than that of fused silica cladding which means that $\Delta n_{\text {eff }}$ will increase with the rising of the temperature and the red shift of dip wavelength can be observed [28]. In addition, the rising and declining of temperature will give rise to the expansion and shrink of the fiber length and further lead to the red and blue shift of the dip wavelength. This can be explained by Equation (3). The mathematical statistics between the temperature and the dip wavelength are implemented and the result is displayed in Figure $5 \mathrm{~b}$. The red line represents the linear fit in the case of temperature increasing and blue line shows that of temperature decreasing. The relationship of temperature and dip wavelength shows good linearity in the process of both temperatures rising and declining and the adjusted $\mathrm{R}$ square values $\left(\mathrm{R}^{2}\right)$ are both 0.99. The slope of temperature increasing is calculated to be $68 \mathrm{pm} /{ }^{\circ} \mathrm{C}$ and $69 \mathrm{pm} /{ }^{\circ} \mathrm{C}$ slope is achieved when temperature decreases. The results are better than that reported in Reference [29] which can be attributed to the different ring core size of the applied fiber. 
(a)

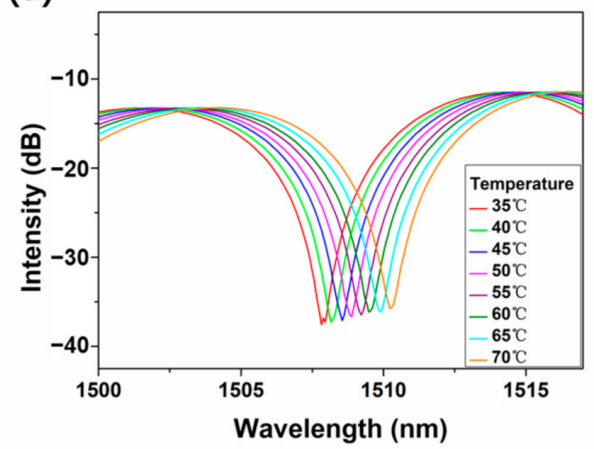

(b)

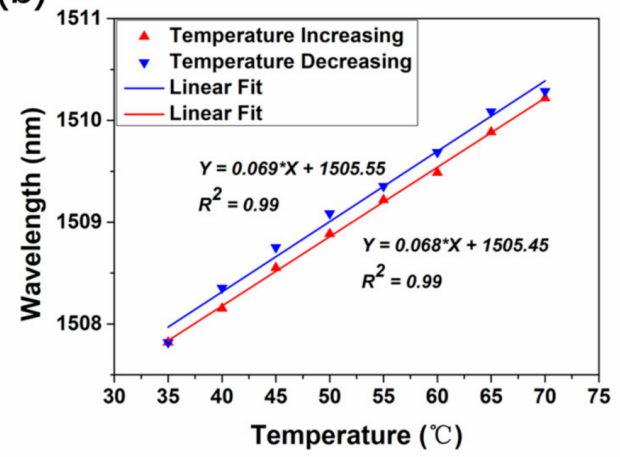

Figure 5. The MZI interference spectra at different temperatures ranging from $35^{\circ} \mathrm{C}$ to $70{ }^{\circ} \mathrm{C}$ with the interval of $5^{\circ} \mathrm{C}$ (a) and the mathematical statistics of temperature and dip wavelength with procedure of temperature increasing and decreasing $(\mathbf{b})$.

The statistics of temperature and interference intensity are also conducted which is depicted in Figure 6. The dip intensity shows very slight alteration as the temperature changes. The linear fit gives the slope of $0.051 \mathrm{~dB} /{ }^{\circ} \mathrm{C}$ which means that the intensity of interference dip at around $1508 \mathrm{~nm}$ is insensitive to thermal effect.

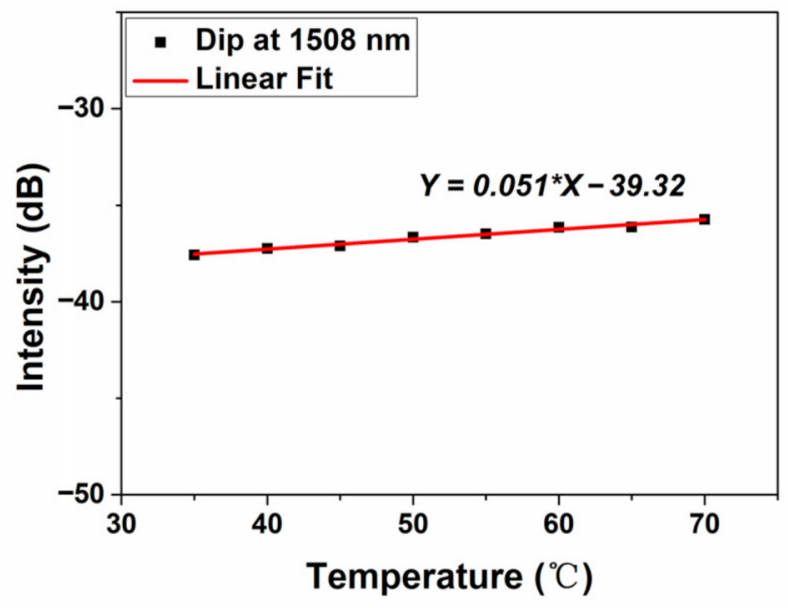

Figure 6. The relationship between interference dip intensity and temperature change at the dip wavelength of around $1508 \mathrm{~nm}$.

The MZI can also be used for RI sensing because the excited cladding modes induced by NCF are sensitive to surrounding RI and the results are shown in Figure 7. Unlike the FC mode and RC mode which transmit around the center of RCF, cladding modes will be reflected on the interface of fiber cladding and air. Thus, cladding modes can be sensitive to the variation of surrounding materials which can easily tune both the amplitude and phase of the cladding modes. The interference spectra will display corresponding alteration according to the change of external environment. Figure 7 a presents the sensing results with different values of RI ranging from 1.33 to 1.38 with the interval of 0.01 at room temperature $\left(25^{\circ} \mathrm{C}\right)$. With the step increasing of surrounding RI, the interference spectra show blue shift which can be explained by Equation (3). In addition, the change of external RI values can also lead to the amplitude change of cladding modes which gives rise to the alteration of interference intensity as shown in Equation (1). Figure $7 \mathrm{~b}$ displays the relationship between RI and the dip intensity. The experiment is repeated and the error bars are signed as black triangle. The RI sensitivity is calculated to be $182.07 \mathrm{~dB} / \mathrm{RIU}$ with the linearity of 0.98 . Compared to the dip intensity change induced by thermal effect, which is $0.051 \mathrm{~dB} /{ }^{\circ} \mathrm{C}$ shown in Figure 6, that influenced by RI is obviously much more 
remarkable. This makes it possible for direct demodulation of RI with the dip intensity by ignoring the weak influence generated by thermal effect. Figure 8 depicts the linear relation between RI and dip wavelength and the slope is calculated to be $-31.44 \mathrm{~nm} / \mathrm{RIU}$. The temperature hence can also be demodulated by using the linear equations between wavelength shift with the variation of RI and temperature:

$$
\begin{gathered}
\Delta \lambda=0.069 \times \Delta T+(-31.44) \times \Delta R I \\
\Delta T=\frac{1}{0.069}(\Delta \lambda+31.44 \times \Delta R I)
\end{gathered}
$$

where $\Delta \lambda$ means the wavelength shift, $\Delta T$ is the change of temperature and $\Delta R I$ represents that of RI.

(a)

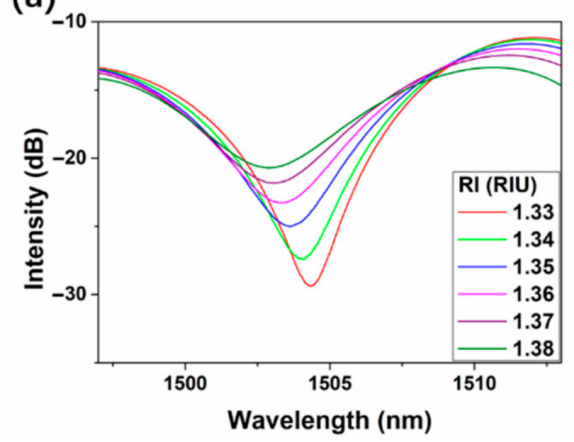

(b)

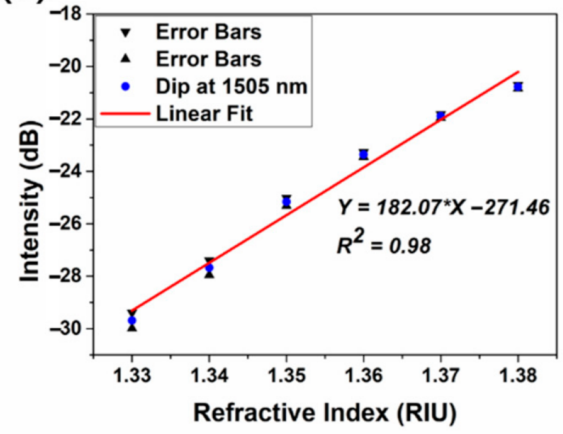

Figure 7. The MZI interference spectra with different RIs ranging from 1.33 to 1.38 with the interval of 0.01 (a) and the relationship between RI and the intensity of interference dips (b).

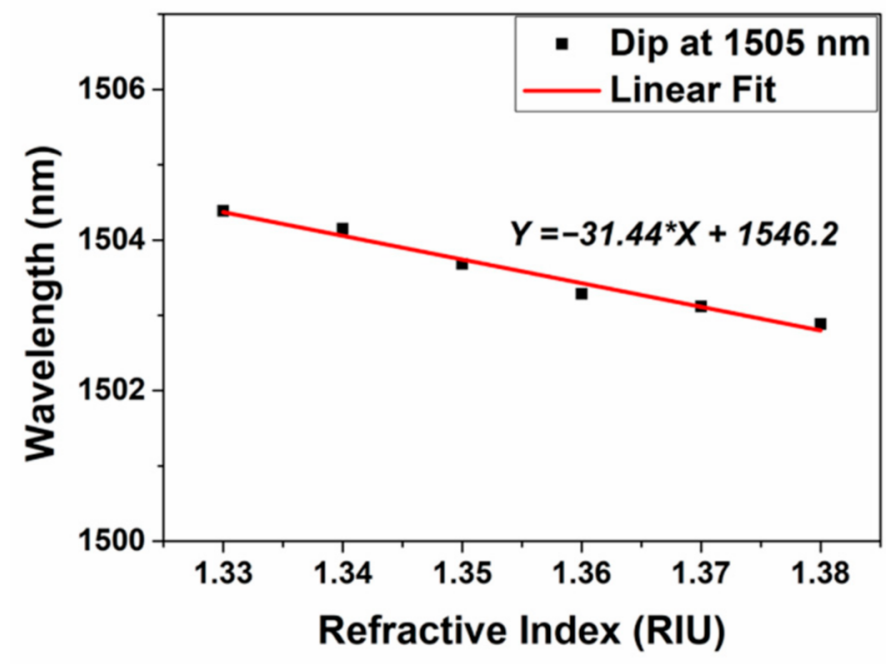

Figure 8. The mathematical statistics of RI and wavelength shift at the dip of around $1505 \mathrm{~nm}$.

\section{Discussion}

Although the influence of thermal effect on interference dip intensity is slight $\left(0.051 \mathrm{~dB} /{ }^{\circ} \mathrm{C}\right)$ in the temperature range from $35^{\circ} \mathrm{C}$ to $70{ }^{\circ} \mathrm{C}$ based on our measurement, large temperature variation can still result in a nonnegligible impact on dip intensity which will lead to a relatively large error on the demodulation of RI. Thus, in our experiment, the dual demodulation of temperature and RI will be confined in a finite temperature range (within $20^{\circ} \mathrm{C}$ variation) to make sure the measurement error be controlled within reasonable limits. Future work can be done to seek solution of such demodulation method that enables sensor 
application within large measurement range, such as optimizing fiber structure or utilizing material assistance.

\section{Conclusions}

In conclusion, the MZI fiber sensor based on SMF-NCF-RCF-SMF structure is successfully achieved. The experiments on temperature and RI sensing are conducted and dual demodulation of temperature and RI can be realized with the temperature variation under $20^{\circ} \mathrm{C}$ and $\mathrm{RI}$ resolution of 0.01 ranging from 1.33 to 1.38 . The maximum sensitivity of temperature sensing is $69 \mathrm{pm} /{ }^{\circ} \mathrm{C}$ and that of RI sensing reaches $182.07 \mathrm{~dB} / \mathrm{RIU}$ and $-31.44 \mathrm{~nm} / \mathrm{RIU}$ with the intensity and wavelength demodulation methods, respectively. The proposed MZI sensor has advantages of cost-effective source, ease of fabrication and high sensitivity for both temperature and RI sensing. In addition, based on the cladding modes modulation, the MZI sensor reveals good potential for diverse applications, such as relative humidity sensing and $\mathrm{pH}$ sensing assisted with chemical materials. Future works will be conducted to extend its applications on variously environmental monitoring.

Author Contributions: Conceptualization, W.Y.; Methodology, W.Y.; Validation, W.Y.; Formal Analysis, W.Y.; Investigation, W.Y.; Writing-Original Draft Preparation, W.Y.; Writing-Review \& Editing, C.Y.; Visualization, W.Y.; Supervision, C.Y.; Project Administration, C.Y. All authors have read and agreed to the published version of the manuscript.

Funding: This work is supported by National Natural Science Foundation of China 61971372 and 152113/17E B-Q60D from HK RGC GRF.

Institutional Review Board Statement: Not applicable.

Informed Consent Statement: Not applicable.

Data Availability Statement: Not applicable.

Acknowledgments: We like to acknowledge the technical support provided by Photonics Research Center, The Shenzhen Institute of The Hong Kong Polytechnic University, Shenzhen, China.

Conflicts of Interest: The authors declare no conflict of interest.

\section{References}

1. Culshaw, B. Optical Fiber Sensor Technologies: Opportunities and-Perhaps-Pitfalls. J. Lightw. Technol. 2004, 22, 39-50. [CrossRef]

2. Li, E.; Wang, X.; Zhang, C. Fiber-optic temperature sensor based on interference of selective higher-order modes. Appl. Phys. Lett. 2006, 89, 091119. [CrossRef]

3. Wen, X.; Ning, T.; Bai, Y.; Li, C.; Li, J.; Zhang, C. Ultrasensitive temperature fiber sensor based on Fabry-Perot interferometer assisted with iron V-groove. Opt. Express 2015, 23, 11526-11536. [CrossRef] [PubMed]

4. Zhang, F.; Xu, X.; He, J.; Du, B.; Wang, Y. Highly sensitive temperature sensor based on a polymer-infiltrated Mach-Zehnder interferometer created in graded index fiber. Opt. Lett. 2019, 44, 2466-2469. [CrossRef]

5. Hernaez, M.; Acevedo, B.; Mayes, A.G.; Melendi-Espina, S. High-performance optical fiber humidity sensor based on lossy mode resonance using a nanostructured polyethylenimine and graphene oxide coating. Sens. Actuators B Chem. 2019, 286, 408-414. [CrossRef]

6. Yuan, W.-H.; Qian, H.; Liu, Y.; Wang, Z.; Yu, C. Highly Sensitive Temperature and Humidity Sensor Based on Carbon NanotubeAssisted Mismatched Single-Mode Fiber Structure. Micromachines 2019, 10, 521.

7. Liang, W.; Huang, Y.; Xu, Y.; Lee, R.K.; Yariv, A. Highly sensitive fiber Bragg grating refractive index sensors. Appl. Phys. Lett. 2005, 86, 151122. [CrossRef]

8. Quan, M.; Tian, J.; Yao, Y. Ultra-high sensitivity Fabry-Perot interferometer gas refractive index fiber sensor based on photonic crystal fiber and Vernier effect. Opt. Lett. 2015, 40, 4891-4894. [CrossRef]

9. Ahsani, V.; Ahmed, F.; Jun, M.B.G.; Bradley, C. Tapered Fiber-Optic Mach-Zehnder Interferometer for Ultra-High Sensitivity Measurement of Refractive Index. Sensors 2019, 19, 1652. [CrossRef] [PubMed]

10. Paixao, T.; Araujo, F.; Antunes, P. Highly sensitive fiber optic temperature and strain sensor based on an intrinsic Fabry-Perot interferometer fabricated by a femtosecond laser. Opt. Lett. 2019, 44, 4833-4836. [CrossRef]

11. Yi, L.; Changyuan, Y. Highly stretchable hybrid silica/polymer optical fiber sensors for large-strain and high-temperature application. Opt. Express 2019, 27, 20107-20116. [CrossRef] 
12. Dong, S.; Dong, B.; Yu, C.; Guo, Y. High Sensitivity Optical Fiber Curvature Sensor Based on Cascaded Fiber Interferometer. J. Lightw. Technol. 2018, 36, 1125-1130. [CrossRef]

13. Marrujo-Garcia, S.; Hernandez-Romano, I.; Torres-Cisneros, M.; May-Arrioja, D.A.; Minkovich, V.P.; Monzon-Hernandez, D. Temperature-independent curvature sensor based on in-fiber Mach-Zehnder interferometer using hollow-core fiber. J. Lightw. Technol. 2020, 38, 4166-4173. [CrossRef]

14. Zhao, Q.; Qian, J.; An, Q.; Du, B. Speedy fabrication of free-standing layer-by-layer multilayer films by using polyelectrolyte complex particles as building blocks. J. Mater. Chem. 2009, 19, 8448-8455. [CrossRef]

15. Semwal, V.; Gupta, B.D. Highly sensitive surface plasmon resonance based fiber optic pH sensor utilizing rGO-Pani nanocomposite prepared by in situ method. Sens. Actuators B Chem. 2019, 283, 632-642. [CrossRef]

16. Wang, T.; Yasukochi, W.; Korposh, S.; James, S.W.; Tatam, R.P.; Lee, S.-W. A long period grating optical fiber sensor with nano-assembled porphyrin layers for detecting ammonia gas. Sens. Actuators B Chem. 2016, 228, 573-580. [CrossRef]

17. Xu, B.; Huang, J.; Xu, X.; Zhou, A.; Ding, L. Ultrasensitive NO Gas Sensor Based on the Graphene Oxide-Coated Long-Period Fiber Grating. ACS Appl. Mater. Interfaces 2019, 11, 40868-40874. [CrossRef]

18. Yao, Q.; Ren, G.; Xu, K.; Zhu, L.; Khan, H.; Mohiuddin, M.; Khan, M.W.; Zhang, B.Y.; Jannat, A.; Haque, F.; et al. 2D Plasmonic Tungsten Oxide Enabled Ultrasensitive Fiber Optics Gas Sensor. Adv. Opt. Mater. 2019, 7, 1901383. [CrossRef]

19. Meng, H.; Shen, W.; Zhang, G.; Tan, C.; Huang, X. Fiber Bragg grating-based fiber sensor for simultaneous measurement of refractive index and temperature. Sens. Actuators B Chem. 2010, 150, 226-229. [CrossRef]

20. Madrigal, J.; Barrera, D.; Sales, S. Refractive Index and Temperature Sensing Using Inter-Core Crosstalk in Multicore Fibers. J. Lightw. Technol. 2019, 37, 4703-4709. [CrossRef]

21. Liao, C.R.; Chen, H.F.; Wang, D.N. Ultracompact Optical Fiber Sensor for Refractive Index and High-Temperature Measurement. J. Lightw. Technol. 2014, 32, 2531-2535. [CrossRef]

22. Fan, R.; Ma, Q.; Li, L.; Zhuo, Y.; Shen, J.; Ren, Z.; Chen, H.; Peng, B. Liquid Level and Refractive Index Double-Parameter Sensor Based on Tapered Photonic Crystal Fiber. J. Lightw. Technol. 2020, 38, 3717-3722. [CrossRef]

23. Zhang, X.-Y.; Yu, Y.-S.; Zhu, C.-C.; Chen, C.; Yang, R.; Xue, Y.; Chen, Q.-D.; Sun, H.-B. Miniature End-Capped Fiber Sensor for Refractive Index and Temperature Measurement. IEEE Photon. Technol. Lett. 2014, 26, 7-10. [CrossRef]

24. Wang, X.; Wang, S.; Jiang, J.; Liu, K.; Zhang, P.; Wu, W.; Liu, T. High-accuracy hybrid fiber-optic Fabry-Perot sensor based on MEMS for simultaneous gas refractive-index and temperature sensing. Opt. Express 2019, 27, 4204-4215. [CrossRef] [PubMed]

25. Lu, H.; Yue, Y.; Du, J.; Shao, L.; Wu, T.; Pan, J.; Hu, J. Temperature and liquid refractive index sensor using P-D fiber structure-based Sagnac loop. Opt. Express 2018, 26, 18920-18927. [CrossRef]

26. Yin, B.; Wu, S.; Wang, M.; Liu, W.; Li, H.; Wu, B.; Wang, Q. High-sensitivity refractive index and temperature sensor based on cascaded dual-wavelength fiber laser and SNHNS interferometer. Opt. Express 2019, 27, 252-264. [CrossRef]

27. Dong, Y.; Xiao, S.; Wu, B.; Xiao, H.; Jian, S. Refractive Index and Temperature Sensor Based on D-Shaped Fiber Combined With a Fiber Bragg Grating. IEEE Sens. J. 2019, 19, 1362-1367. [CrossRef]

28. Lu, P.; Men, L.; Sooley, K.; Chen, Q. Tapered fiber Mach-Zehnder interferometer for simultaneous measurement of refractive index and temperature. Appl. Phys. Lett. 2009, 94, 131110. [CrossRef]

29. Li, X.; Chen, N.K.; Xi, L.; Zhang, H.; Zhang, X.; Zhang, W.; Tang, X. Micro-fiber Mach-Zehnder interferometer based on ring-core fiber. Opt. Express 2019, 27, 34603-34610. [CrossRef] [PubMed] 